\title{
Resiliencia de jóvenes deportistas en función del carácter individual o colectivo de su práctica
}

\section{Resilience of young athletes depending on the individual or collective character of their practice}

\author{
Arkaitz Zubizarreta Cortadi ${ }^{1}$, Silvia Arribas Galagarraga ${ }^{2}$ y Izaskun Luis de $\operatorname{Cos}^{3}$ \\ 1 Profesor asociado del Dpto. De Didáctica de la Expresión Musical, Plástica y Corporal. EHU/UPV. San Sebastián (España) \\ 2 Titular de Universidad. Dpto: Didáctica de la Expresión Musical, Plástica y Corporal. EHU/UPV. San Sebastián (España) \\ 3 Prof. Adjunta Dpto: Didáctica de la Expresión Musical, Plástica y Corporal. EHU/UPV. San Sebastián (España)
}

\begin{abstract}
Resumen: El objetivo de este estudio fue determinar los niveles de resiliencia con sus dos dimensiones, "Aceptación de uno mismo y de la vida" (AUM) y "Competencia Personal" (CP),en participantes de deportes de carácter individual y colectivo. Se realizaron comparaciones inter e intragrupo y en función del nivel competitivo y sexo. Un total de 435 deportistas (300 hombres y 135 mujeres) cumplimentaron un cuestionario socio-demográfico y La Escala de Resiliencia (Wagnild y Young, 1993). Los resultados señalan que quienes practican deportes de carácter individual obtienen valores medios de resiliencia y de la dimensión CP superiores a los obtenidos por deportistas que lo hacen de forma colectiva $(\mathrm{p}<0.005)$. Respecto al sexo, en la dimensión AUM, las chicas presentan medias menores que los chicos $(\mathrm{p}<0.005)$. En cuanto al nivel competitivo, la tendencia es que a mayor nivel competitivo, mayor puntuación media de resiliencia $(\mathrm{p}<0.05)$.Los resultados dibujan la imagen del deportista resiliente como un chico joven practicante de deporte de carácter individual y de alto nivel competitivo. Palabras clave: Resiliencia, deportes carácter individual y colectivo, nivel competitivo, sexo.
\end{abstract}

\section{Introducción}

El afán de superación, la tolerancia, fomentar el respeto a los demás o a las reglas, la responsabilidad, la disciplina, cooperación, entre otros muchos valores (Berengúi y Garcés 2007) son aspectos deseables a incorporar en el proceso de enseñanza-aprendizaje, y el deporte se presenta como elemento potencialmente facilitador en el ámbito pedagógico y formativo.Sin embargo, si la enseñanza deportiva se aborda sin una orientación adecuada, puede fomentar actitudes no deseables entre quienes lo practican. Según Burgués, Llanes y Guiu (2012), el deporte, en función de su carácter, individual o colectivo, o la modalidad practicada, puede aportar diferentes tendencias.Sin embargo, como explica García (2016), la tendencia actual respecto a las investigaciones realizada en deportes de carácter colectivo y de carácter individual está

Dirección para correspondencia [Correspondence address]: Arkaitz Zubizarreta Cortadi. Dpto. De Didáctica de la Expresión Musical, Plástica y Corporal. EHU/UPV. San Sebastián. Plaza Oñati, no3. 20018 Guipúzcoa (Espańa). E-mail: arkaitz.zubizarreta@ehu.eus
Abstract: The objective of this study was to determine the levels of resilience with its two dimensions, "acceptance of self and life" (AUM) and "individual competence" (CP), in individual and collective sports participants. Comparisons depending on competitive level and sex, as well as, intragroup and intergroup comparisons were performed. A total of 435 athletes (300 men and 135 women) completed a socio-demographic questionnaire and the Resilience Scale (Wagnild and Young, 1993). The results indicate that those who practise individual sports obtain average values of resilience and the $\mathrm{CP}$ dimension higher than those obtained by athletes who do so collectively ( $\mathrm{p}<0.005$ ). According to sex, in the AUM dimension, girls have lower averages than boys $(\mathrm{p}<0.005)$. As for the competitive level, the trend is that at a higher competitive level, higher average resilience score $(\mathrm{p}<$ 0.05 ). The results draw the image of the athlete resilient as a young boy practicing sport of individual character and high level competitive. Key words: Resilince, individual and collective sports, competitive level, sex.

equilibrada, siendo escasas las investigaciones comparativas entre ambos (individual o colectivo).

Como postula Méndez (2003), los deportes de carácter colectivo, son más susceptibles de generar actitudes que proporcionen el desarrollo de una serie de valores, facilitados por la expresión y relación con los demás, adquiriendo autoconfianza o el sentimiento de pertenencia a un grupo (Mendez, 2003; Usán, Salavera, Murillo y Megías 2015).Este factor de socialización, ha sido estudiado en profundidad por Martinez et al (2018), quienes concluyeron que a medida que el deportista compite a mayor nivel, la importancia que le da al factor de socialización como razón de la práctica deportiva disminuye.

Así mismo, se puede pensar que los deportes de carácter individual pueden fomentar la necesidad de autonomía, como concluyeron Gené y Latinjak (2014), al estudiar las Necesidades Psicológicas Básicas y la autodeterminación en deportistas de deportes de carácter individual y colectivo, resultando que la necesidad de autonomía en los deportistas individuales era mayor. Reforzando esta línea de investigación, Fernández, 
Contreras, García y González, (2010) descubrieron que los deportistas que más entrenan, en especial en las dimensiones físicas, presentan mayores niveles de autoconcepto.

La resiliencia se encuentra entre los aspectos psicológicos más estudiados durante los últimos ańos. En la actualidad, los investigadores coinciden en definir la resiliencia como, el conjunto de habilidades personales que permiten a un individuo un funcionamiento saludable o la adaptación a un contexto adverso y/o a un acontecimiento disruptivo en la vida diaria (Connor y Davidson, 2003; Luthar, Cicchetti y Becker, 2000; Masten y Obradovic, 2006). Al respecto, Burtona, Pakenhamb y Brown (2010) señalan que la resiliencia es la capacidad que permite enfrentarse, ajustarse o recuperarse del estrés o de la adversidad. Asimismo, Vanistendael (2010, p.11) considera que "la resiliencia es la capacidad que tiene un individuo o un grupo para superar grandes dificultades y crecer". En esa línea se puede decir que la resiliencia no es absoluta, por el contrario, es una capacidad que se encuentra en proceso de construcción durante toda la vida (Vanistendael, 2010). Por su parte, López Torres (2010, p.2) indican que "la resiliencia nunca se adquiere de una vez y para siempre, es importante la existencia permanente e integrada de una red de apoyos sociales, afectivos y materiales".

En la revisión realizada por Secades, y col. (2014), la Resiliencia se considera como una variable multidimensional que incluye atributos psicológicos y disposicionales, tales como competencia, sistemas de apoyo externo y estructura personal (Campbell-Sills, Cohan y Stein, 2006; Connor y Davidson, 2003; Masten, 2001). Esta cualidad se adquiere en un proceso dinámico mediante el cual, el sujeto se adapta adecuadamente a contextos significativamente adversos (Luthar, Cicchetti y Becker, 2000).

En la misma línea Martinek y Hellison, (1997) defienden que la actividad física al ser activa, interactiva y altamente emocional, permite y facilita, explorar y practicar los diferentes rasgos que conforman la resiliencia.

De acuerdo con Lyoka (2011), los programas desarrollados con base en ejercicios pueden aumentar la estabilidad emocional, la autoconfianza, las funciones cognitivas y el trabajo en red social, los cuales son factores de importancia en el desarrollo de la resiliencia. Otra estrategia para promover la resiliencia, es la incorporación de ejercicios de relajación, estiramiento, respiración y meditación (Shelton, 2008; Sood, Prasad, Schroeder y Varkey, 2011; Quiceno, Vinaccia y Remor, 2011). Numerosos programas recreativos también han sido usados como un medio para fomentar la resiliencia. Entre las actividades recreativas más usadas para este fin, se encuentran las actividades deportivas, campamentos, actividades al aire libre, actividades de aventura, actividades de fin de semana, voluntariado en la comunidad, programas de artes escénicas, actividades recreativas de noche, juegos y programas después de clases (Beth, Mezei, Bush y Weinstein,
2012; Ewert y Yoshino,2011; Green, Kleiber y Tarrant, 2000). Los resultados anteriormente señalados también coinciden con las diversas publicaciones realizadas en el último decenio, en las que se menciona como actividades que fomentan la resiliencia, los programas de actividad física, de ejercicio, deporte, o recreación (Leigh y Milgrom 2008; Lyoka, 2011).

Las investigaciones con referencias a la Resiliencia en el ámbito deportivo se han incrementado en los últimos años. LópezSuárez (2014) estudió la relación entre resiliencia y el deporte en jóvenes deportistas, entre 11 y 17 años. Entre las conclusiones halladas destacan que no se observan diferencias significativas en las dimensiones de la escala de resiliencia en función de la edad. Otra diferencia significativa se observa en una de las dimensiones de la Resiliencia (Aceptación de uno mismo y de la vida), los resultados parecen indicar que los jóvenes cuyas edades están comprendidas entre los 11 y los 13 años están más satisfechos con la vida que los jóvenes mayores de 13 ańos. En cuanto a la posible relación entre el tiempo que llevan practicando deporte los sujetos, con sus niveles de resiliencia, no se encontraron diferencias significativas en los ítems ni en las dimensiones de la escala de resiliencia, pero los resultados parecen indicar que los jóvenes que llevan más de un año practicando deporte puntúan significativamente más en la dimensión de Aceptación de uno mismo y de la vida, que los que practican desde hace menos de un año y además considera que su vida es cercana a su ideal.

Por su parte, Barquero (2015), realiza un metaanálisis del efecto de la actividad física en el desarrollo de la resiliencia. encontrado valor significativamente mayor en resiliencia en los grupos experimentales que realizaron algún tipo de actividad física.En otro estudio, Zurita, Castro, Linares y Chacón (2017) concluyeron que hacer deporte, más concretamente el atletismo, aumentaba la resiliencia. Con el fin de profundizar en esta línea de investigación, Chacón-Cuberos et al. (2016) mencionan que sería de gran interés incluir la medición del nivel de ejercicio físico de las actividades que realizan los participantes mediante acelerómetros para conocer la intensidad de la práctica deportiva en función del deporte, modo de práctica y sexo de los participantes.

El estudio de la resiliencia en función del género, es otro aspecto que suscita el interés científico. En diferentes estudios (Palomar y Gómez, 2010; Beightol, Jevertson, Carter, Gray y Gass, 2012) se halló que las mujeres presentaban niveles más altos de resiliencia que los hombres, aunque los propios autores, señalaban la necesidad de más estudio de esta línea de investigación.

El presente estudio aborda la faceta competitiva del deporte, que se caracteriza por la necesidad de afrontar lassensaciones y percepciones fluctuantes que pueden influir en el incrementos de bienestar mental (Fox, 1999; Arruza, Arribas, De Montes, Irazusta, Romero y Cecchini, 2008). Para ello, y teniendo en cuenta queel deporte es considerado de gran potencial para el desarrollo de valores sociales y habilidades 
personales (Jacobs, Knoppers y Webb, 2013), se considera relevante profundizar en el conocimiento de variables que faciliten el desarrollo de respuestas positivas a situaciones de gran estrés y adversidad.

En esta investigación se estudia la resiliencia de los deportistas en función del sexo, el carácter individual o grupal del deporte y su nivel competitivo.

\section{Método}

\section{Diseño}

Se trata de un estudio con diseño ex post-facto, de carácter descriptivo e inferencial. El muestreo se realizó por conveniencia.

\section{Participantes / Muestra(PD)}

Los participantes en este estudio son 435 deportistas de más de 14 años que como mínimo llevan 2 años federados. La muestra está compuesta por 300 hombres y 135 mujeres de edades comprendidas entre los 14 y 44 años ( $M=18,61$; Dt $=4,91)$. La participación por modalidad fue del 37\% para deportes de carácter individual (23\% para atletismo y $14 \%$ para natación) y $63 \%$ para deportes de carácter colectivo (41,6\% para fútbol y $21,4 \%$ para balonmano). La distribución de la muestra en función del ámbito territorial de competición es la siguiente; el 0,9\% de los participantes compite en la misma ciudad, 63\% en Guipúzcoa, 30,6\% en Euskadi y $5,5 \%$ en España (tabla 1 ).

Tabla 1. Distribución de la muestra en función carácter, modalidad deportiva, nivel competitivo y género.

Tabla 1. Caracteristicas de la muestra.

\begin{tabular}{|c|c|c|}
\hline Variable & $\mathrm{N}$ & $\%$ \\
\hline Carácter colectivo & 274 & 63 \\
\hline Futbol & 181 & 41.6 \\
\hline Balonmano & 93 & 21.4 \\
\hline Carácter individual & 161 & 37 \\
\hline Atletismo & 100 & 23 \\
\hline Natación & 61 & 14 \\
\hline \multicolumn{3}{|l|}{ Nivel de competición. } \\
\hline Misma urbe & 4 & 0.9 \\
\hline Guipuzcoa & 274 & 63.0 \\
\hline Euskadi & 133 & 30.6 \\
\hline España & 24 & 5.5 \\
\hline \multicolumn{3}{|l|}{ Sexo } \\
\hline Chica & 135 & 31 \\
\hline Chico & 300 & 69 \\
\hline
\end{tabular}

SPORT TK: Revista Euroamericana de Ciencias del Deporte

\section{Instrumentos de evaluación}

La Escala de Resiliencia (Wagnild y Young, 1993) es uno de los instrumentos más adecuados para la evaluación de la resiliencia en ámbito deportivo deportiva. Estos autores elaboran un instrumento de evaluación que convierte este concepto en medible diferenciando dos factores generales (Competencia Personal y Aceptación de uno mismo y de la vida). Estos dos factores toman en consideración las siguientes características de la resiliencia: satisfacción personal, ecuanimidad, sentirse bien solo, confianza en sí mismo y perseverancia (Lévano, 2005). La primera escala de resiliencia contenía 50 ítems y tras el análisis factorial inicial, la escala se redujo a 25 ítems. En este estudio se ha utilizado la versión validada en Castellano (Ruiz, Marcos, Poveda, Serpa, y Rosado, 2012). Las puntuaciones, oscilan en un rango entre 25 y 175, las puntuaciones mayores de 145 indican un nivel elevado de resiliencia, de 121-145 moderados y menores de 121 indican poca capacidad de resiliencia (Ruiz, De la Vega, Poveda, Rosado y Serpa 2012).

Los alpha para la escala total fueron de $.808 y$ para cada una de las dimensiones fueron las siguientes: Competencia personal.765 y .494 para el factor Aceptación de uno mismo y de la vida.

La escala utilizada ha resultado con un alpha de .845 para la escala total, y .850 para Competencia personal, y .523 para Aceptación de uno mismo y de la vida.

\section{Procedimiento}

El muestreo fue llevado a cabo siguiendo los criterios de conveniencia y siguiendo los dictados de la Comisión de Ética para la Investigación con Seres Humanos (CEISH) de la UPV/EHU (código de la investigación: M10_2017_187 aprobado en el acta 96/2018).Se contactó con las federaciones Guipuzcoanas de Balonmano, Fútbol, Natación y Atletismo,solicitándoles información sobre los 5 clubes Deportivos con mayor número de fichas de la provincia. Una vez recibido el listado, se contactó con cada uno de ellos para explicarles la investigación y el proceso de recogida de datos. Tras recibir las autorizaciones pertinentes, los investigadores se personaron para llevar a cabo la toma de datos.

\section{Análisis de datos}

Tras comprobar la normalidad de los datos, mediante la prueba Kolgorov-Smirnov, la homoestacidad de varianzas con el test de Levene y la linealidad de los datos, se procedió a realizar un estadístico descriptivo para encontrar la media aritmética y su desviación típica (M, DT) en función de las diferentes variables estudiadas. Para analizar en más profundidad la diferencia entre variables, se realizaron diferencia de 
medias mediante prueba T-test y ANOVA de un factor para la variable de más de dos categorías. El análisis de datos se realizó con el programa IBM SPSS v. 24.

\section{Resultados}

En este apartado, inicialmente, se realizaron los análisis descriptivos de la muestra según la variable de la resiliencia y sus dos dimensiones, Aceptación de uno mismo y de la vida (AUM) y Competencia Personal (CP). Como se observa en la tabla 2, los mayores valores en la escala de Resiliencia (rango entre 25 y 175) la muestran los jóvenes que practican deporte individual, 138,12 (Dt=13,62) frente a 133,75 puntos $(\mathrm{Dt}=14,94)$ para los deportes colectivos. Centrándonos en los deportes, la media de los atletas fue de 139,94 seguidos de los futbolistas 136,35 , los nadadores 135,16 y por último nos encontramos con los federados en Balonmano 128,59 (Tabla 2). Todos los grupos presentan valores codificados como moderados en la escala de la Resiliencia (Wagnild y Young, 1993). Respecto a las puntuaciones en función del sexo, las chicas puntúan 134.48 (Dt=14.44) y los chicos 135.77 (Dt=14.69). Observando el nivel competitivo de los participantes, las puntuaciones de quienes compiten en su entorno han sido de 136.75 $(\mathrm{Dt}=27.94)$, las de quienes compiten a nivel provincial (Guipúzcoa) 134.26 (Dt=14.04), a nivel territorial (Comunidad Autónoma Vasca) 136.03 (Dt=14.53) y a nivel estatal (España) 143.92 (Dt=16.59) (Tabla 2).

Una vez realizado el análisis descriptivo, se realizó un análisis estadístico de comparación de medias, mediante Prueba T-test (tabla 3). Los resultados señalan que los deportistas de carácter individual (138.13) obtienen valores medios de resilienciasuperiores que los deportistas de carácter colectivo (133.76), siendo estos estadísticamente significativos $\mathrm{t}(419)=$ -2,987: $\mathrm{P}<0.005$. Además,en la dimensión de Competencia Personal(CP) los deportistas de carácter individual (97.38) obtienen puntuaciones medias mayores que los deportistas de carácter colectivos (93.07) siendo estas diferencias estadísticamente significativas, $\mathrm{t}(419)=0,836: \mathrm{p}<0.005$.

Tabla 2. Puntuación en la escala de Resiliencia de los participantes en función del carácter, género y nivel deportivo.

\begin{tabular}{|c|c|c|c|c|}
\hline & \multicolumn{2}{|c|}{ Resiliencia } & \multirow{2}{*}{$\frac{\text { AUM }}{\mathrm{M}}$} & \multirow{2}{*}{$\frac{\mathrm{CP}}{\mathrm{M}}$} \\
\hline & M & DT & & \\
\hline Carácter colectivo & 133.76 & 14.94 & 40.68 & 93.08 \\
\hline Futbol & 136.35 & 13.86 & 41.49 & 94.86 \\
\hline Balonmano & 128.6 & 15.73 & 39.07 & 89.53 \\
\hline Carácter individual & 138.13 & 13.62 & 40.75 & 97.38 \\
\hline Atletismo & 139.95 & 13.87 & 41.54 & 98.40 \\
\hline Natación & 135.17 & 12.78 & 39.46 & 95.71 \\
\hline \multicolumn{5}{|l|}{ Sexo } \\
\hline Chica & 138.48 & 14.44 & 39.67 & 94.81 \\
\hline Chico & 135.77 & 14.69 & 41.16 & 94.60 \\
\hline \multicolumn{5}{|l|}{ Nivel deportivo } \\
\hline Misma urbe & 136.75 & 27.94 & 42.75 & 94.00 \\
\hline Guipúzcoa & 134.26 & 14.04 & 40.73 & 93.53 \\
\hline Euskadi & 136.03 & 14.53 & 40.05 & 95.98 \\
\hline España & 143.92 & 16.59 & 43.50 & 100.42 \\
\hline
\end{tabular}

Valores expresados en media y desviación típica (M, DT).

Respecto al sexo, se observa que no hay diferencias significativas en los valores de resiliencia entre chicos $(134,48)$ y chicas $(135,77)$. Sin embargo la dimensión Aceptación de uno mismo y de la vida (AUM), las chicas (39.66) obtienen puntuaciones medias menores que los chicos (41.16) siendo estas diferencias estadísticamente significativas $\mathrm{t}(419)=2,637$ : $\mathrm{p}<0.005$.

Tabla 3. Pruebas T-test de diferencia de medias entre carácter deportivo y sexo.

\begin{tabular}{|c|c|c|c|c|c|c|c|c|c|c|c|c|c|}
\hline \multirow[t]{2}{*}{ Variable } & \multicolumn{4}{|c|}{ Resiliencia } & \multicolumn{5}{|c|}{ AUM } & \multicolumn{4}{|c|}{$\mathrm{CP}$} \\
\hline & $\mathrm{N}$ & M & DT & $\mathrm{t}(\mathrm{gl})$ & sig & M & DT & $\mathrm{t}(\mathrm{gl})$ & sig & M & DT & $\mathrm{t}(\mathrm{gl})$ & sig \\
\hline \multicolumn{14}{|c|}{ Carácter deportivo } \\
\hline Colectivo & 266 & 133.76 & 14.94 & \multirow{2}{*}{ 2,99 (419) } & \multirow{2}{*}{0,003} & 40.68 & 5.28 & \multirow{2}{*}{$0,12(419)$} & \multirow{2}{*}{0,902} & 93.07 & 11.19 & \multirow{2}{*}{$0,84(419)$} & \multirow{2}{*}{0,000} \\
\hline Individual & 155 & 138.13 & 13.62 & & & 40.74 & 5.68 & & & 97.38 & 10.25 & & \\
\hline \multicolumn{14}{|l|}{ Sexo } \\
\hline chico & 291 & 134.48 & 14.44 & \multirow{2}{*}{0,84 (419) } & \multirow{2}{*}{0,403} & 41.16 & 5.31 & \multirow{2}{*}{$2,64(419)$} & \multirow{2}{*}{0,009} & 94.59 & 11.13 & \multirow{2}{*}{0,18 (419) } & \multirow{2}{*}{0,857} \\
\hline chica & 130 & 135.77 & 14.69 & & & 39.66 & 5.56 & & & 94.80 & 10.86 & & \\
\hline
\end{tabular}

Para analizar la relación entre la resiliencia y sus dimensiones con el nivel competitivo de los deportistas, se han realizado comparaciones de medias mediante ANOVA de un factor. Como se observa en la tabla 4. En relación a variable de la res- iliencia, se puede observar que los valores obtenidos muestran una tendencia a mayor nivel competitivo, mayor puntuación media de resiliencia, siendo los deportistas de nivel estatal los que mayor puntuación obtienen (143.92).Estas diferencias 
en los valores obtenidos, son estadísticamente significativas $F(3,420)=3,406: \mathrm{p}<0.05$.

En el análisis de las dimensiones de la resiliencia, los datos muestran que los valores fluctúan en función del nivel competitivo, siendo los de mayor nivel competitivo los que alcanzan un mayor nivel de AUM. Cabe señalar que las diferencias existentes entre nivel competitivo son estadísticamente significativas $(F(3,420)=2,961: \mathrm{p}<0.05)$.
Respectoa la dimensión CP los deportistas de mayor nivel competitivo, obtienen niveles de más altos $(100,41)$, observando como a medida que aumenta el nivel competitivo los deportistas obtienen mayores valores en CP. La relación que existe entre las variables es estadísticamente significativa $(F(3,420)=3,792: \mathrm{p}<0.05)$.

Tabla 4. ANOVA de diferencia de medias entre nivel competitivo.

\begin{tabular}{lcccccccccc}
\hline Variable & \multicolumn{2}{c}{ Misma urbe } & \multicolumn{2}{c}{ Guipúzcoa } & \multicolumn{2}{c}{ Euskadi } & \multicolumn{2}{c}{ España } & \multirow{2}{*}{$\mathrm{F}(3,420)$} & $\mathrm{p}$ \\
& $\mathrm{M}$ & $\mathrm{DT}$ & $\mathrm{M}$ & $\mathrm{DT}$ & $\mathrm{M}$ & $\mathrm{DT}$ & $\mathrm{M}$ & $\mathrm{DT}$ & & \\
& 136,75 & 27,94 & 134,25 & 14,04 & 136,03 & 14,53 & 143,91 & 16,59 & 3,406 & 0.018 \\
Resiliencia & 42,75 & 9,06 & 40,73 & 5,22 & 40,05 & 5,44 & 43,50 & 6,21 & 2,961 & 0.032 \\
AUM & 94,00 & 19,47 & 93,52 & 10,59 & 95,97 & 10,95 & 100,41 & 12,88 & 3,792 & 0.011 \\
CP & & & & & & & &
\end{tabular}

\section{Discusión}

Debido al aumento de la importancia del deporte y la actividad física como elemento formativo, sobre todo en edades de desarrollo, el objeto de este estudio fue analizar la resiliencia de los deportistas en función del carácter individual o grupal de la competición deportiva, el sexo y su nivel competitivo. En ese camino se analizó si el carácter deportivo se relacionaba con la Resiliencia. Los resultados muestran que quienes practican modalidades de carácter individual son más resilientes que quienes practican deportes de carácter colectivo. En esta línea, la investigación de Chacón-Cuberos et al. (2016), confirma los mayores valores de resiliencia en Esquiadores que en balonmanistas y futbolistas.

$\mathrm{Al}$ analizarlasdimensiones que componen la resiliencia se observa que la Competencia Personal es el componente más fuerte. Sospechando que el carácter individual del deporte practicado tiene gran influencia en la buena percepción de la competencia personal del deportista. A su vez, Zurita, Castro, Linares, Chacon-Cuberos (2018) al encontrar valores mayores de resiliencia en atletas sugieren "que al tratarse de un deporte individual, la capacidad de autoeficacia y resistencia al malestar son más valoradas, repercutiendo en un incremento en los índices de capacidad de sobreponerse a la adversidad" (p. 571).

Respecto a las diferencias de Resiliencia en función del sexo, al analizar el constructo en su totalidad, no se observan diferencias. Sin embargo,las chicas, tienen valores significativamente menores que los chicos en la dimensión de Aceptación de uno mismo y de la vida. Estos resultados confirman el estudio de Zurita et. al. (2017) en el que los hombres valoran mejor su competencia personal que las mujeres. A su vez estos resultados contradicen el estudio de Palomar y Gómez (2010) y Beightol (2008), quienes encontraron que las mujeres presentaban niveles más altos de resiliencia que los hombres. Aunque coincidimos con el propio Beightol (2008), quien señala la necesidad de mayor estudio de esta línea de investigación.

En cuanto al nivel deportivo, cabe destacar que los deportistas de mayor nivel competitivo tienenvalores significativamente mayores de resiliencia. Esta significación toma más fuerza en la dimensión de Competencia personal. Estos resultados pueden ser debidos, como argumentan Ryckman y Hamel (1993, citado por Sánchez-Alcaraz et al. 2018) a la edad y la experiencia: a menor categoría, puede haber mayor dependencia de los compañeros-amigos de práctica, a su vez a mayor nivel competitivo, los sujetos gozan de mayor autonomía e independencia.

\section{Conclusión}

Una vez realizados los análisis y la discusión de los resultados podemos concluir que la resiliencia es una variable poco estudiada pero de gran importancia en el ámbito deportivo. Los resultados dibujan la imagen del deportista resiliente como un chico joven practicante de deporte de carácter individual $\mathrm{y}$ de alto nivel competitivo

Estos resultados han de tomarse con cautela puesto que los deportes de carácter individual elegidos para este estudio requieren mayor exigencia física que los de carácter colectivo. No obstante se insiste en la necesidad de realizar estudios que aborden esta temática. 


\section{Referencias bibliográficas}

1. Arruza, J., Arribas, S., De Montes, L. G., Irazusta, S., Romero, S., \& Cecchini, J. (2008). Repercusiones de la duración de la actividad físicodeportiva sobre el bienestar psicológico. Revista Internacional De Medicina y Ciencias De La Actividad Fisica y Del Deporte/International Journal of Medicine and Science of Physical Activity and Sport, 8(30), 171-183.

2. Beightol, J., Jevertson, J., Carter, S., Gray, S., \& Gass, M. (2012). Adventure education and resilience enhancement. Journal of Experiential Education, 35(2), 307-325.

3. Beth, M., Mezei, B., OTS, A., Weinstein PhD, M., \& Bush PhD, J. (2012). Engagement, environment, and skill development: An exploration of the summer activity experiences of at-risk middle school youth.

4. Burgués, P. L., Llanes, J. M., \& Guiu, G. F. (2012). Juegos deportivos y emociones. propiedades psicométricas de la escala GES para ser aplicada en la educación física y el deporte. Revista De Investigación Educativa, 31(1), 151-165.

5. Burton, N. W., Pakenham, K. I., \& Brown, W. J. (2010). Feasibility and effectiveness of psychosocial resilience training: A pilot study of the READY program. Psychology, Health \& Medicine, 15(3), 266-277.

6. Campbell-Sills, L., Cohan, S. L., \& Stein, M. B. (2006). Relationship of resilience to personality, coping, and psychiatric symptoms in young adults. Behaviour Research and Therapy, 44(4), 585-599.

7. Chacon Borrego, F., Padial Ruz, R., Yedra Clements, S., Breton Prats, S., Cepero Gonzalez, M., \& Zurita Ortega, F. (2017). Relationship between the academic achievement and the self-concept in young basketball players in extracurricular national competition. Sport TkRevista Euroamericana De Ciencias Del Deporte, 6(2), 75-80.

8. Connor, K. M., \& Davidson, J. R. (2003). Development of a new resilience scale: The Connor-Davidson resilience scale (CD-RISC). Depression and Anxiety, 18(2), 76-82.

9. Cuberos, R. C., Giráldez, V. A., Zagalaz, J. C., Sánchez, M. L. Z., \& García, D. C. (2016). Estudio relacional de la práctica deportiva en escolares según el género. SPORT TK-Revista EuroAmericana De Ciencias Del Deporte, 5(1), 85-92.

10. Ewert, A., \& Yoshino, A. (2011). The influence of short-term adventurebased experiences on levels of resilience. Journal of Adventure Education and Outdoor Learning, 11(1), 35-50.

11. Fernández, J. G., Contreras, O. R., García, L. M., \& González Villora, S. (2010). Autoconcepto físico según la actividad físicodeportiva realizada y la motivación hacia ésta. Revista Latinoamericana De Psicología, 42(2), 251-263.

12. Fox, K. R. (1999). The influence of physical activity on mental wellbeing. Public Health Nutrition, 2(3a), 411-418.

13. Fox, K. R. (1999). The influence of physical activity on mental wellbeing. Public Health Nutrition, 2(3a), 411-418.

14. García Bertó, R. (2016). Revisión sobre las diferencias de las variables psicológicas en deportes individuales vs. deportes de equipo.

15. García Secades, X., Molinero, O., Ruíz Barquín, R., Salguero, A., Vega, R. d. 1., \& Márquez, S. (2014). La resiliencia en el deporte: Fundamentos teóricos, instrumentos de evaluación y revisión de la literatura. Cuadernos De Psicología Del Deporte, 14(3), 83-98.

16. Gené, P., \& Latinjak, A. T. (2014). Relación entre necesidades básicas y autodeterminación en deportistas de élite. Cuadernos De Psicología Del Deporte, 14(3), 49-56.

17. Gil, R. B., \& de los Fayos, Enrique J Garcés. (2007). Valores en el deporte escolar: Estudio con profesores de educación física. Cuadernos De Psicología Del Deporte, 7(2), 89-104

18. Green, G. T., Kleiber, D. A., \& Tarrant, M. A. (2000). The effect of an adventure-based recreation program on development of resiliency in low income minority youth. Journal of Park \& Recreation Administration, $18(3)$
19. Jacobs, F., Knoppers, A., \& Webb, L. (2013). Making sense of teaching social and moral skills in physical education. Physical Education and Sport Pedagogy, 18(1), 1-14.

20. Jacobs, F., Knoppers, A., \& Webb, L. (2013). Making sense of teaching social and moral skills in physical education. Physical Education and Sport Pedagogy, 18(1), 1-14.

21. Leigh, B., \& Milgrom, J. (2008). Risk factors for antenatal depression, postnatal depression and parenting stress. BMC Psychiatry, 8(1), 24

22. Lévano, A. C. S. (2005). Métodos e instrumentos para medir la resiliencia: Una alternativa peruana. Liberabit, 11, 41.

23. López-Suárez, M. (2014). Relación Entre Satisfacción Con La Vida y Satisfacción Con El Deporte y En Jóvenes Deportistas,

24. Luthar, S. S. (2006). Resilience in development: A synthesis of research across five decades.

25. Luthar, S. S., Cicchetti, D., \& Becker, B. (2000). The construct of resilience: A critical evaluation and guidelines for future work. Child Development, 71(3), 543-562.

26. Lyoka, P. (2011). Impact of intermittent physical exercises on mental health of some police officers in nkonkobe district, south africa: Sport psychology. African Journal for Physical Health Education, Recreation and Dance, 17(Special issue 1), 665-674.

27. Martinek, T. J., \& Hellison, D. R. (1997). Fostering resiliency in underserved youth through physical activity. Quest, 49(1), 34-49.

28. Martínez, B. J. S., Marín, D. M., Ibáñez, J. C., Pérez, F. J. G., Herrera, R. C., \& García, J. D. (2018). Motivos de la práctica de pádel en relación a la edad, el nivel de juego y el género. Practice motives in padel players regarding age, level and gender.

29. Masten, A. S. (2001). Ordinary magic: Resilience processes in development. American Psychologist, 56(3), 227.

30. Masten, A. S., \& Obradović, J. (2006). Competence and resilience in development. Annals of the New York Academy of Sciences, 1094(1), 13-27.

31. Ortega, F. Z., Sánchez, M. C., Manrique, M. L., \& Cuberos, R. C. (2016). Resiliencia, un elemento de prevención en actividad física. Sportis, Scientific Journal of School Sport, Physical Education and Psychomotricity, 3(1), 50-62.

32. Palomar Lever, J., \& Gómez Valdez, N. E. (2010). Desarrollo de una escala de medición de la resiliencia con mexicanos (RESI-M). Interdisciplinaria, 27(1), 7-22.

33. Portzky, M., Wagnild, G., De Bacquer, D., \& Audenaert, K. (2010) Psychometric evaluation of the dutch resilience scale RS-nl on 3265 healthy participants: A confirmation of the association between age and resilience found with the swedish version. Scandinavian Journal of $\mathrm{Ca}$ ring Sciences, 24(s1), 86-92.

34. Quiceno, J. M., Vinaccia, S., \& Remor, E. (2011). Programa de potenciación de la resiliencia para pacientes con artritis reumatoide. Revista De Psicopatología y Psicología Clínica, 16(1), 27-47.

35. Ruiz, R., de la Vega, R., Poveda, J., Rosado, A., \& Serpa, S. (2012) Análisis psicométrico de la escala de resiliencia en el deporte del fútbol. Revista De Psicología Del Deporte, 21(1).

36. Secades, X. G., Molinero, O., Barquín, R. R., Salguero, A., De la Vega, R., \& Márquez, S. (2014). La resiliencia en el deporte: Fundamentos teóricos, instrumentos de evaluación y revisión de la literatura. Cuadernos De Psicología Del Deporte, 14(3), 83-92.

37. Shelton, D. (2008). Translating theory into practice: Results of a 2 year trial for the LEAD programme. Journal of Psychiatric and Mental Health Nursing, 15(4), 313-321.

38. Sood, A., Prasad, K., Schroeder, D., \& Varkey, P. (2011). Stress management and resilience training among department of medicine faculty: A pilot randomized clinical trial. Journal of General Internal Medicine, 26(8), 858-861. 
39. Torres, V. L. (2010). Educación y resiliencia: Alas de la transformación social. Revista Electrónica" Actualidades Investigativas En Educación, 10(2), 1-14

40. Vanistendael, S. (2010). Derechos del niño y resiliencia. Bruselas: Cuadernos De La BICE,
41. Villasana, M., Alonso-Tapia, J., \& Ruiz, M. A. (2017). Personal factors underlying resilience in adolescence: Cross-cultural validity of the prince-embury model. The Spanish Journal of Psychology, 20

42. Wagnild, G. M., \& Young, H. M. (1993). Development and psychometric evaluation of the resilience scale. Journal of Nursing Measurement.
SPORT TK: Revista Euroamericana de Ciencias del Deporte

ISSN edición web: 2340-8812 / vol. 8, n. ${ }^{\circ} 2$ / Murcia / Julio 2019 / Págs. 73-80
Resiliencia de jóvenes deportistas en función del carácter individual o colectivo de su.. SPORT TK, 8(2), 73-80 